\title{
G Research Square \\ The Effectiveness Of Prolotherapy For Recalcitrant Medial Tibial Stress Syndrome: A Prospective Case Series
}

\section{Nat Padhiar ( $\sim$ nat.padhiar@londonsportswise.co.uk)}

Centre for Sports \& Exercise Medicine, Queen Mary, University of London https://orcid.org/0000-00020352-4534

\section{Mark Curtin}

Centre for Sports \& Exercise Medicine, Queen Mary University of London

\section{Osama Aweid}

Centre for Sports \& Exercise Medicine, Queen Mary University of London

\section{Bashaar Awied}

Imperial College London Faculty of Medicine

\section{Dylan Morrissey}

Centre for Sports \& Exercise Medicine, Queen Mary University of London

\section{Otto Chan}

The London Independent Hospital

\section{Peter Malliaras}

Monash University Faculty of Medicine Nursing and Health Sciences

\section{Tom Crisp}

Cntre for Sports \& Exercise Medicine, Queen Mary University of London

\section{Research}

Keywords: Prolotherapy, medial tibial stress syndrome, injection, exercise induced leg pain, dextrose

Posted Date: November 11th, 2020

DOI: https://doi.org/10.21203/rs.3.rs-33075/v2

License: (c) (i) This work is licensed under a Creative Commons Attribution 4.0 International License. Read Full License

Version of Record: A version of this preprint was published at Journal of Foot and Ankle Research on April 16th, 2021. See the published version at https://doi.org/10.1186/s13047-021-00453-z. 


\section{Abstract}

Background: Medial tibial stress syndrome (MTSS) is one of the most common lower leg injuries in sporting populations. It accounts for between $6 \%-16 \%$ of all running injuries, and up to $53 \%$ of lower leg injuries in military recruits. Various treatment modalities are available with varying degree of success. In recalcitrant cases, surgery is often the only option.

Objective: To evaluate whether ultrasound-guided injection of $15 \%$ dextrose for treatment of recalcitrant Medial Tibial Stress Syndrome decreases pain and facilitates a return to desired activity levels for those who may otherwise be considering surgery or giving up sport.

Design: Prospective case series

Setting: Private specialist Centre

Patients: Eighteen patients: fifteen male and three female; (mean age=31.2 years) with MTSS were referred from sports injury clinics across the UK, having failed all available conservative treatment.

Intervention: An ultrasound-guided sub-periosteal injection of $15 \%$ dextrose was administered by the same clinician (NP) along the length of the symptomatic area. Typically, $1 \mathrm{~mL}$ of solution was injected per $\mathrm{cm}$ of symptomatic area.

Main Outcome Measures: Pain was assessed using a 10-cm visual analogue scale (VAS) at baseline, short-term, medium-term (mean 18 weeks) and long-term (mean one year) follow-up. Symptom resolution and return to activity were measured using a Likert scale at medium and long-term follow-up.

Results: Patients reported a significant $(p<0.01)$ reduction in median VAS pain score at medium and longterm follow-up compared to baseline. Median improvement per patient was 4.5/10. Patients rated their condition as 'much improved' at medium-term follow-up and median return to sport score was 'returned to desired but not pre-injury level' at medium-term and long-term follow-up. No adverse events were reported.

Conclusions: Ultrasound-guided 15\% dextrose prolotherapy injection has a significant medium-term effect on pain in MTSS. This benefit may be maintained long-term. More robust trials are required to validate these findings.

Clinical Relevance: Clinicians should consider the use of ultrasound-guided injection of $15 \%$ dextrose as a viable treatment option to reduce pain and aid return to activity for patients with recalcitrant Medial Tibial Stress Syndrome.

\section{Introduction}

Medial tibial stress syndrome (MTSS) is one of the most common lower leg injuries in sporting populations[1]. It accounts for between $6 \%-16 \%$ of all running injuries[2], and up to $53 \%$ of lower leg 
injuries in military recruits[3].

The diagnosis is reliably based on detail history and physical examination with pain provoked on palpation of the lower $1 / 3^{\text {rd }}$ of medial tibial [4].

In the early stages of the condition, pain tends to occur at the beginning of exercise, may diminish as activity proceeds, and recurs at the end[2, 5, 6]. Usually, pain resolves over a variable period of rest[7]. However, as the condition progresses, pain may occur constantly throughout exercise[8], at rest and at night, causing significant distress and affecting quality of life[2, 5, 9].

Medial tibial stress syndrome is predominantly managed conservatively. Treatment modalities which have shown potential benefit include extracorporeal shockwave therapy (ESWT), ultrasound therapy, iontophoresis, ice massage, periosteal pecking $(10,11,12,13)$, non-steroidal anti-inflammatory drugs (NSAIDs), stretching and orthotics[14], and modification of biomechanical factors. However, a systematic review by Winters et al [15] showed no evidence for the effect of any intervention in treating MTSS. In recalcitrant cases surgery is considered, although it significantly reduced pain in $72 \%$ of those treated, only $41 \%$ of patients returned to sport at their previous level [16].Although common, the pathophysiological process underlying MTSS remains uncertain. A popular theory is that excessive muscular traction could lead to inflammation of the periosteum, causing chronic periostitis $(8,17,18)$. However, histological studies have found scant evidence of inflammation[19,20,21]. More recently, it has been suggested that MTSS be classified as a point on a continuum of bone stress reaction, which can be assessed using magnetic resonance imaging (MRI)(22). Beck (1998) proposed that repetitive loading during sustained weight-bearing activity may lead to strain-related periosteal remodelling due to tibial bending, which provokes stress injury at the point of maximum bending(23)

Winters el al (2019) found linear microcracks in the biopsies of athletes with MTSS with no repair reaction, suggesting unrepaired microdamage as an underlying pathophysiology (24).

Proliferative injection therapy (prolotherapy) has been used clinically since the late $19^{\text {th }}$ century and has been mentioned in medical journals since at least 1937[25]. The rationale behind prolotherapy is that injecting proliferants, such as hypertonic glucose solution, into damaged connective tissue, initiates inflammation, which leads to a healing cascade resulting in fibroplasia, deposition of new collagen and tissue hypertrophy(26) Animal studies have reported collagen proliferation, increased bone-ligament-bone junction strength and ligament mass with prolotherapy injections compared to controls(27). The periosteum is richly innervated with nociceptive nerve fibres(28), therefore in MTSS it is possible that a prolotherapy injection may reduce pain by disrupting these sensory fibres as a result of the direct osmotic shock action of hypertonic dextrose on cells local to the injection site(26).

Trials of prolotherapy have found it to be beneficial in the treatment of lateral epicondylopathy(29), osteitis pubis(30), plantar fasciopathy(31), Achilles tendinopathy(32) and recalcitrant coccygodynia(33). There is currently no published literature investigating the use of prolotherapy in the management of MTSS. A pilot study of this project reported a median pain reduction of $50 \%$ at medium-term follow-up 
(34). The present study was a continuation of that pilot study and enabled longer-term treatment effects to be established. With current management options for recalcitrant MTSS showing inconsistent or unsatisfactory results, investigation of this novel treatment was necessary.

This prospective case series study sought to evaluate whether ultrasound-guided injection of hypertonic dextrose decreases pain and facilitates a return to desired activity levels for those who may otherwise be considering surgery or giving up sport. We hypothesise that prolotherapy improves pain and facilitates return to sport at a desired level.

\section{Patients And Methods}

\section{Recruitment}

Sports physicians, orthopaedic surgeons, podiatrists, podiatric surgeons and physiotherapists working in sports injury clinics who were known to the main 2 authors (NP, TAC) across the UK were contacted via email and invited to refer patients with painful, recalcitrant MTSS not responding to other conservative treatment modalities, whom they felt might benefit from the trial intervention. (Figure.1.)Failed previous conservative treatment included assessing and addressing any lower limb issues both proximal and distal, with strength, flexibility, proprioception, balance, ultrasound therapy, ESWT, acupuncture, needling and Graston fascia release, rest, ice, NSAIDs (non-steroidal anti-inflammatory drugs) and correction of biomechanics (e.g. foot orthoses, brace and taping for control of foot pronation).

Inclusion criteria were patients with persistent, painful MTSS assessed and confirmed by the lead clinician (NP). Exclusion criteria were previous periosteal surgery for MTSS, previous or current tibial stress fracture or contraindications to the intervention such as pregnancy or anticoagulant therapy.

Patients were assessed by the lead clinician and the diagnosis of MTSS was confirmed by a history of exercise induced pain over the posteromedial border of the middle to distal third of the tibia, a positive SPT (Shin Palpation Test - palpation tenderness over the painful middle and distal thirds of the medial tibia) both at rest and following exercise, and MRI changes showing periosteal or bone marrow oedema.

Ethical approval was granted by the Queen Mary University of London Ethics Review Board (QMREC2009/22). Participants provided signed written informed consent. Subsequently, a detailed medical history and biographical data were taken and an examination performed.

\section{Intervention and injection procedure}

The injection procedure is very simple and within the expertise of all clinicians who are used to injection techniques for musculo-skeletal pathology. It is primarily performed in the out-patient department (OPD) and does not require a local anaesthetic even though local anaesthetic is used to dilute $50 \%$ glucose down to $15 \%$. In some cases where pain tolerance is poor, it can be performed under a general anaesthetic. In this study all subjects were treated in OPD. The target area for the purpose of needle placement is the area anterior to deep crural fascia (Figs. 1A and 1B.) along the medial tibia. The skin 
overlying the most painful area of the tibia was marked with an indelible marker pen and then cleansed using alcoholic chlorhexidine ( $2 \%$ chlorhexidine gluconate, $70 \%$ isopropyl alcohol). An ultrasound scanner (USS) (Siemens AG, Berlin, Germany) was used to guide and confirm needle position. The needle is introduced under real-time USS from the most proximal end of the site of pain (knee end) to the most distal (ankle end) (Figs 2A \& 2B). The spinal needle ( $0.7 \mathrm{~mm}$ diameter $x 90 \mathrm{~mm}$ length, Becton, Dickinson and Company LLC, Franklin Lakes, New Jersey, US) was positioned parallel along the medial tibia in the area anterior to deep crural fascia (Fig.3). Needle introducer was removed with the needle in place. 15\% dextrose solution was slowly infiltrated, approximately $1 \mathrm{~mL}$ of solution per $1 \mathrm{~cm}$ along the whole length of the area of pain. In some case where the length was longer than the spinal needle, second entry point was made following the same protocol as above. After the injection, the area was cleansed, dressed, ice packs applied for 2 minutes and knee high compression socks (Fig.4.) (Bauerfeind AG, Zeulenroda-Triebes, Germany) were fitted. Patients were advised to continue wearing them for up to four weeks, removing them at night. Patients were advised to take relative rest for three days and given advice for simple flexibility exercises and a graded return to physical activity. Patients were followed up one week later to monitor progress and address any concerns or questions.

\section{Outcome Measures}

The patients' 'average pain', defined as the most pervasive severity of pain throughout a 24-hour period, was measured using a 10-cm visual analogue scale (VAS). The VAS is sensitive[35], reliable, valid and responsive for measuring pain in other common musculoskeletal conditions, such as patellofemoral pain syndrome[36]. Pain was assessed in this way at 0 (baseline), 1, 2 and 4 weeks after the injection, and at medium-term (mean 18 weeks, range 13-36 weeks) and long-term (mean 52 weeks, range 47-74 weeks) follow-up to assess the patients' response to the intervention.

A Likert[37] symptom resolution scale was used to measure the subjective degree of recovery at mediumterm and long-term follow-up compared to baseline. There are six possible outcome scores for the Likert scale: 1- completely recovered, 2- much improved, 3- somewhat improved, 4- no change, 5- worse, 6much worse. Treatment was classed as a success in patients who rated themselves as 'completely recovered' or 'much improved', reflecting the method of previous authors(38). Other scores were considered treatment failure. Categorical scales such as the Likert are sensitive indicators of clinical trial end points(37).

Return to sport was assessed with a five-point activity scale at medium-term and long-term follow-up: 1not active at all, 2- no return to sport, 3- returned to sport at an unsatisfactory lower level, 4- returned at desired but not pre-injury level, 5- returned at pre-injury level. Although not reported in other literature, the aim of treatment was to enable patients to return to their desired sports at pre-injury levels. All other activity scores were considered treatment failure.

\section{Statistical Analysis}


Statistical analyses were performed using SPSS for Mac version 19.0.0 (IBM, New York, NY, US).

Statistical significance was set at a $p$-value less than 0.05. The Shapiro-Wilk test was used to evaluate normality of the distribution of data. Distribution of data was negatively skewed and not normally distributed (Shapiro-Wilk = 0.009) therefore appropriate non-parametric tests were performed to evaluate the changes in pain levels.

Median values and interquartile ranges were calculated to compare baseline and follow-up data for VAS average pain scores, Likert symptom resolution and return to sport scores.

Friedman's non-parametric test was used to compare within-patient treatment response over time. Posthoc Wilcoxon signed-rank tests with Bonferroni corrections were performed to determine VAS average pain response to treatment over five paired time periods (baseline - 4 weeks, baseline - 18 weeks, 4 - 18 weeks, baseline - 52 weeks, 4 weeks - 52 weeks). The Bonferroni corrected alpha value was $(p<0.01)$.

\section{Results}

Twenty five legs of eighteen patients were injected. Of those twenty five, seven legs were injected a second time. For the purposes of data collection, each patient was treated as a whole case rather than individual legs.

On average, patients reported that post-injection pain took three days to settle. Side effects were not asked for specifically, but there were no self-reported adverse events following injection.

The mean age and duration of symptoms for the patients are summarised in Table.1. Two patients had undergone surgical fasciotomy for diagnosed chronic compartment syndrome on their affected limbs.

Table.1. Mean age and duration of symptoms.

\begin{tabular}{|l|l|}
\hline Characteristic & \\
\hline Male & $15(83 \%)$ \\
\hline Female & $3(17 \%)$ \\
\hline Mean age & 34 \\
\hline Mean symptom duration & 52 \\
\hline Mean BMI & 25.9 \\
\hline
\end{tabular}

$\mathrm{BMI}=$ Body Mass Index

\section{Descriptive Analysis}

Data for eighteen patients was available for analysis of treatment effect to medium-term and fifteen patients to long-term follow-up. Three patients were lost to follow-up and these data points were not imputed but omitted from long-term analysis. 
Medians and interquartile ranges for the three outcome measures are displayed in Table.2. Change in median VAS average pain score over time is displayed in Figure.5.

2 patients had complete resolution of symptoms $(11 \%, \mathrm{n}=18)$ at 18 weeks and also at 1 year. 8 patients $(33 \%, n=18)$ were much improved at 18 weeks and $3(20 \%, n=15)$ at 1 year. 6 patients $(33 \%, n=18)$ were somewhat improved at 18 weeks and $3(20 \%, n=15)$ at 1 year. There was no change in symptom resolution in 2 patients $(11 \%, n=18)$ at 18 weeks and 7 patients $(47 \%, n=15)$ at 1 year. There were no patients who were worse or made worse following prolotherapy. (Table.3.)

Table.2. Median values and interquartile ranges for VAS average pain, symptom resolution and level of activity.

\begin{tabular}{|c|c|c|c|}
\hline & & Median & Interquartile range \\
\hline VAS average pain score $(\mathrm{cm})$ & Baseline & 7.5 & $6-8$ \\
\hline \multirow{4}{*}{$\begin{array}{c}0 \text { - no pain } \\
10 \text { - worst pain imaginable }\end{array}$} & 2 weeks & 2 & $1-3.75$ \\
\hline & 4 weeks & 3 & $1-4$ \\
\hline & 18 weeks & 3 & $2-4$ \\
\hline & 52 weeks & 3 & $2-4.5$ \\
\hline Likert symptom resolution score & 18 weeks & 2 & $2-3$ \\
\hline $\begin{array}{c}1 \text { - completely recovered } \\
6 \text { - much worse }\end{array}$ & 52 weeks & 3 & $2-4$ \\
\hline Activity level & 18 weeks & 4 & $3-5$ \\
\hline $\begin{array}{c}1-\text { not active at all } \\
5 \text { - active at pre-injury level }\end{array}$ & 52 weeks & 4 & $3-4.5$ \\
\hline
\end{tabular}

Table.3. Summary of symptom resolution and activity level outcomes. 


\begin{tabular}{|l|l|l|}
\hline & 18 weeks $(\mathrm{n}=18)$ & 1 year $(\mathrm{n}=15)$ \\
\hline Likert symptom resolution & \multicolumn{2}{|l|}{} \\
\hline Completely recovered & $2(11.11 \%)$ & $2(13.33 \%)$ \\
\hline Much improved & $8(33.33 \%)$ & $3(20 \%)$ \\
\hline Somewhat improved & $6(33.33 \%)$ & $3(20 \%)$ \\
\hline No change & $2(11.11 \%)$ & $7(46.67 \%)$ \\
\hline Worse & $0(0 \%)$ & $0(0 \%)$ \\
\hline Much worse & $0(0 \%)$ & $0(0 \%)$ \\
\hline \multicolumn{2}{|l|}{} \\
\hline Activity level & \multicolumn{2}{|l|}{} \\
\hline Returned at pre-injury level & $5(27.78 \%)$ & $4(26.67 \%)$ \\
\hline Returned at desired but not pre-injury level & $5(27.78 \%)$ & $4(26.67 \%)$ \\
\hline Returned to sport at an unsatisfactory lower level & $8(44.44 \%)$ & $6(40 \%)$ \\
\hline No return to sport & $0(0 \%)$ & $1(6.67 \%)$ \\
\hline Not active at all & $0(0 \%)$ & $0(0 \%)$ \\
\hline
\end{tabular}

5 patients $(28 \%, n=18)$ returned to pre-injury level return to sport at 18 weeks and 4 patients $(27 \%, n=15)$ at 1 year. 5 patients $(28 \%, n=18)$ returned to desired but not pre-injury level return to sport at 18 weeks and 4 patients $(27 \%, n=15)$ at 1 year. 8 patients $(44 \%, n=18)$ returned to sport at unsatisfactory level at 18 weeks and 6 patients $(40 \%, n=15)$ at 1 year. 1 patient failed to return to any level of sport or activity and elected to undergo surgery. (Table.3.).

\section{Statistical Analysis}

Friedman's test of the VAS average pain scores to medium-term (mean 18 weeks) reported a Chi-squared value of $30.3, p<0.001$. There was a statistically significant difference between the mean pain ranks over time. Therefore, post-hoc analysis was warranted. Friedman's test was also significant to long-term follow-up, reporting a Chi-squared value of $27.5, p=<0.001$.

Changes in VAS average pain score rank and $p$-values for the five paired time periods are reported in Table.4. VAS average pain decreased for 16 patients from baseline to both 4 weeks $(p<0.001)$ and 18 weeks follow-up $(p<0.001)$. One patient had an increase in pain at 4 weeks which subsequently decreased at 18 weeks follow-up and another patient reported no change at 4 weeks and 18 weeks. Between 4 weeks and 18 weeks after the injection, pain increased in 7 patients (38.8\%), decreased in 5 $(27.7 \%)$, and remained the same in 6 patients (33.3\%) $(p=0.405)$. Between baseline and 52 weeks, pain decreased in 13 patients (86.7\%), remained the same in one patient $(6.7 \%)$ and increased in one patient $(6.7 \%)(p=0.001)$. Between 4 weeks and 52 weeks, pain decreased in five patients $(33.3 \%)$, remained the same in 4 patients $(26.7 \%)$ and increased in 6 patients $(40 \%)(p=0.322)$.

Table.4. Rank data and significance for the Wilcoxon signed-rank tests for VAS average pain. A negative rank represents an improvement in a patient's pain over that time period. A positive rank represents worsening pain over that time period. 


\begin{tabular}{|c|c|c|c|}
\hline $\begin{array}{c}\text { Time period } \\
(\mathrm{n}=18)\end{array}$ & Ranks & $\mathbf{N}$ & $\boldsymbol{p}$-value \\
\hline $\begin{array}{c}\text { Baseline - 4 weeks } \\
\text { Nogative ranks }\end{array}$ & 16 & $<0.001$ \\
& Ties & 1 & \\
\hline Baseline - 18 weeks & Negative ranks & 16 & $<0.001$ \\
$(\mathrm{n}=18)$ & Positive ranks & 0 & \\
& Ties & 2 & \\
\hline 4 weeks - 18 weeks & Negative ranks & 5 & 0.405 \\
& Positive ranks & 7 & \\
& Ties & 6 & \\
\hline Baseline - 52 weeks & Negative ranks & 13 & 0.001 \\
& Positive ranks & 1 & \\
& Ties & 1 & \\
\hline 4 weeks - 52 weeks & Negative ranks & 5 & 0.322 \\
$(\mathrm{n}=15)$ & Positive ranks & 6 & \\
& Ties & 4 & \\
\hline
\end{tabular}

After Bonferroni corrections were applied, the change in VAS average pain from baseline to 4,18 and 52 weeks follow-up were statistically significant $(p<0.01)$. The changes in VAS average pain from 4 to 18 weeks and 4 to 52 weeks were not significant $(p>0.01)$

\section{Discussion}

Pain was significantly reduced $(\mathrm{p}<0.01)$ over short, medium, and long-term compared to baseline, with only two patients not reporting a reduction in VAS average pain over these time periods (Table.4). The median VAS average pain score improved by 4.5 points at medium and long-term follow-up compared to baseline, equivalent to a $60 \%$ reduction in pain (Table.2).

However, a wider range of pain scores were seen at long-term (Figure.5), in addition to a larger p-value for average pain reduction, possibly due to the lower statistical power at this stage of follow-up. There was also a trend for improvement in pain to recede from 4 weeks post-injection, suggesting that pain control 
is most effective within the first month after administration. This is a potential window of opportunity to implement other conservative management options that could facilitate long-term pain control.

At 18 weeks (medium-term) follow-up, ten patients (55.5\%) reported their MTSS as 'completely recovered' or 'much improved', indicating treatment success at this stage. Six patients $(40 \%)$ fulfilled these criteria at 52 weeks follow-up, which signifies a potential longer-term decline in treatment effect .(Table.4.)

Five patients $(27.7 \%)$ reported a return to sport at pre-injury levels at medium-term follow-up and four patients $(26.7 \%)$ at long-term follow-up. Treatment seems to have a limited effect on this outcome measure. However, ten patients (55.5\%) were active at a desired level or more at medium-term follow-up and eight (53.3\%) at long-term follow-up, which suggests we may have been too stringent in selecting the treatment success criteria for this outcome measure. 1 patient elected to undergo surgery, as at 1 year there was no improvement and failed to return to sport.

\section{Conclusion}

Dextrose prolotherapy injection was well tolerated and significantly improved pain at short term and, its effect retained in the long term. Adequate symptom resolution and return to sport was achieved at medium-term follow-up and return to sport was maintained long-term. This prospective study consisting of selective recalcitrant cases therefore suggests that prolotherapy has a significant effect on short, medium and long-term pain reduction.

Prolotherapy is an effective treatment modality in the management of recalcitrant MTSS and even though 1 patient elected to undergo surgery, this study was not designed to assess whether there will be reduction in the number of patients that may require surgery. This study did not involve any histological sampling to explain the effect on the tissue. We postulate that $15 \%$ glucose acts as an osmotic and chemical irritant (dehydrating cells) along with damage to tissue through needling causing local trauma and bleeding. This provokes cascade of inflammation, proliferation, and re-modelling. The rationale behind prolotherapy is that injecting proliferants, such as hypertonic glucose solution, into damaged connective tissue, initiates inflammation, which leads to a healing cascade resulting in fibroplasia, deposition of new collagen and tissue hypertrophy[26]. It is also possible that prolotherapy improves the mechanical advantage with improved stability of deep crural fascia junction with medial tibia. Animal studies have reported collagen proliferation, increased bone-ligament-bone junction strength and ligament mass with prolotherapy injections compared to controls[27]. The reduction in pain may be due periosteum being richly innervated with nociceptive nerve fibres[28], therefore in MTSS it is possible that a prolotherapy injection may reduce pain by disrupting these sensory fibres as a result of the direct osmotic shock action of hypertonic dextrose on cells local to the injection site[26]. It is also worth debating, even though there is no evidence, whether, if the sensory fibers are disrupted, could this, apart from the positive effects on pain and function short-term, produce adverse effects long term e.g. disturbed bone remodeling potentially leading to stress fractures/worsening MTSS/osteoporosis? 
However, in sport, benefit of pain free phase can be effectively used to provide a platform for early rehabilitation and a window of opportunity to plan return to sport or physical activity specific rehabilitation.

\section{LIMITATIONS}

As the first study investigating this novel injection technique, the use of a non-blinded, case series design without randomisation or a control group was a pragmatic choice given the difficulties recruiting participants. Evidence of treatment effect is therefore limited. Despite results showing a significant treatment effect on pain, the mechanism by which it exerts this effect cannot be determined, and therefore the possibility of a placebo effect cannot be excluded in addition to the possibility these patients may have improved with time without treatment.

Patients were only included in the study if they had MRI evidence of periosteal changes or bone marrow oedema. MTSS is a clinical pain syndrome with unknown aetiology. The evidence suggest that periosteal and bone marrow oedema are absent in the majority (i.e. 56\%) of clinically diagnosed cases with MTSS (39). Furthermore, periosteal and bone marrow oedema are often present in healthy asymptomatic athletes $(40,41)$ which suggests it is not an adequate characteristic to identify those with MTSS. As a result, we may have missed relevant cases with MTSS with negative MRI findings.

Given that there are currently no validated scales to specifically assess treatment outcomes in EILP patients, we adapted Likert scales to measure symptom resolution and return to sport. Although Likert scales are sensitive indicators of clinical trial end points[37], the lack of specificity could at least partly explain the limited treatment effect for these outcomes. Since this study was conducted there are 3 validated patient outcome scores are now available $(42,43,44)$, one is specific for MTSS (42).

\section{CONTEXT}

The current literature on the effects of prolotherapy on chronic musculoskeletal conditions is limited. Two case-series investigating the efficacy of dextrose prolotherapy injections at reducing pain in other chronic musculoskeletal conditions reported mean reductions of 5.3 and 5.0 respectively on 10-cm VAS pain scales[29, 30]; one study followed patients up at 16 weeks, the other at final treatment consultation. A double-blind randomised controlled trial reported a significant mean pain decrease of 4.6 on a $10-\mathrm{cm}$ pain scale at 16 weeks from baseline[28]. Pain change in the control group was not significant. These results are comparable to our study, where a 4.5-point reduction of pain was reported at 18 weeks follow-up. One study observed patients to long-term follow-up (mean 11.8 months), reporting mean VAS pain reduction of 5.3 points compared to baseline[30]. Pain reduction was maintained long-term in Ryan et al's study, which is similar to the 4-point VAS average pain reduction in our study.

\section{FUTURE STUDIES}

Future studies require more robust methodologies including larger participant numbers, a control group or crossover design, randomisation and, if possible, blinding to improve validity of the results. Adverse 
effects should be explicitly sought to permit a more thorough treatment profile to be compiled.

VAS average pain data from follow-up and one and two weeks post-injection appear of limited application regarding treatment direction, with the decision to re-inject patients with suboptimal symptomatic response coming at four weeks or later. Follow-up at four weeks then monthly may allow better monitoring of the treatment response over the medium- to long-term and facilitate the decision to re-inject for those patients whose symptoms have not responded as well as predicted.

With some patients requesting a second injection, it may be prudent to administer more than one prolotherapy injection per symptomatic leg, reflecting the protocols of other studies where injections were administered weekly or monthly, ranging from 2 to 12 injections over the study period[29,32,33], or until complete resolution of symptoms or no improvements were seen[30, 32].

\section{Declarations}

Acknowledgements: The authors express thanks to the patients, and both the administrative and clinical teams at the London Independent Hospital and at Centre for Sports \& Exercise Medicine, Queen Mary, University of London, for their assistance and patience. Special thanks to Dr Paul Jones and Sister Mel Pritchard for all her help in ensuring smooth running of the study.

Funding: There are no funding issues. This research received no specific grant from any funding agency in the public, commercial, or not-for-profit sectors. relevant, registered) have been explained.

Availability of data and materials: There are no issues concerning this as strict confidentiality was observed and subjects were anonymised. The authors affirm that this manuscript is an honest, accurate, and transparent account of the study being reported; that no important aspects of the study have been omitted; and that any discrepancies from the study as planned (and, if relevant, registered) have been explained.

Authors' contributions: This was a collaborative study. NP and TC contributed to conception and design of the study, interpretation of the data, as well as drafting and writing the manuscript and final approval. $\mathrm{MC}, \mathrm{OA}$ and BA collected the data, contributed to manuscript writing. DM and PM contributed to manuscript writing and statistical and Data analysis. OC developed the injection protocol and technique and, contributed to manuscript writing. All authors agree to be accountable for all aspects of the work. All authors read and approved the final manuscript.

Ethics approval \& Consent to participate: Queen Mary, University of London (QMUL) granted approval of this study. QMREC2009/22 - A prospective study to assess the effectiveness of prolotherapy in the management of unresponsive and painful Medial Tibial Stress Syndrome (MTSS). All subjects provided written informed consent prior to participating in the study.

Consent for publication: Participants and QMUL were aware that findings will be disseminated at conferences and results will be published. 
Competing interests: None declared. The authors disclose no conflict or competing of interest, they did not receive any financial payments or other benefits from any commercial entity related to the subject of this article. No outside source of funds was involved in the preparation of or editing of the manuscript.

\section{References}

1. Orava S, Puranen J. Athletes' leg pains. British journal of sports medicine. 1979;13:92-97.

2. Kortebein P M, Kaufman K R, Basford J R, et al. Medial tibial stress syndrome. Medicine and science in sports and exercise. 2000;32:S27.

3. Yates $B$, White $S$. The incidence and risk factors in the development of medial tibial stress syndrome among naval recruits. The American journal of sports medicine. 2004;32:772-780.

4. Winters M, Bakker EWP, Moen MH, Barten CC, Teeuwen R, Weir A. Medial tibial stress syndrome can be diagnosed reliably using history and examination. Br J Sports Med. 2018;52:1-6.

5. Edwards P H, Wright M L, Hartman J F. A practical approach for the differential diagnosis of chronic leg pain in the athlete. The American journal of sports medicine. 2005;33:1241-1249.

6. Moore M. Shin splints. Diagnosis, management, prevention. Postgraduate medicine. 1988;83:199200, 203-195, 208-110.

7. Moen M H, Tol J L, Weir A, et al. Medial tibial stress syndrome. Sports medicine. 2009;39:523-546.

8. Mubarak S J, Gould R N, Lee Y F, et al. The medial tibial stress syndrome a cause of shin splints. The American journal of sports medicine. 1982;10:201-205.

9. Cosca D D, Navazio F. Common problems in endurance athletes. Am Fam Physician. 2007;76:237244.

10. Newman P, Waddington G, Adam R. Shockwave treatment for medial tibial stress syndrome; a randomized double blind sham-controlled pilot trial. J Sci Med Sports. 2017 Mar;20(3):220-224.

11. Gomez Garcia S, Ramon Rona S, Gomez Tinoco MC, et al. Shockwave treatment for medial tibial stress syndrome in military cadets: A single-blind randomized controlled trial. Int J Surg. 2017;46:102-109.

12. Smith W, Winn F, Parette R. Comparative study using fourmodalities in shinsplint treatments. $J$ Orthop Sports Phys Ther.1986;8(2):77-80.

13. Robertson ME. The relative effectiveness of periosteal peckingcombined with therapeutic ultrasound compared to therapeuticultrasound in the treatment of medial tibial stress syndrome typell. Faculty of Health at the Durban Institute of Technology,Durban, South-Africa.

14. Loudon J K, Dolphino M R. Use of foot orthoses and calf stretching for individuals with medial tibial stress syndrome. Foot \& ankle specialist. 2010;3:15-20.

15. Winters M, Eskes M, Weir A, Moen MH, Backx FJ, Bakker EW. Treatment of medial tibial stress syndrome: a systematic review. Sports Med. 2013;43(12):1315-1333. doi:10.1007/s40279-013-00870 
16. Yates B, Allen M J, Barnes M R. Outcome of surgical treatment of medial tibial stress syndrome. The Journal of Bone \& Joint Surgery. 2003;85:1974-1980.

17. Holder L E, Michael R H. The specific scintigraphic pattern of" shin splints in the lower leg": concise communication. Journal of nuclear medicine: official publication, Society of Nuclear Medicine. 1984;25:865-869.

18. Michael R H, Holder L E. The soleus syndrome A cause of medial tibial stress (shin splints). The American journal of sports medicine. 1985;13:87-94.

19. Bhatt R, Lauder I, Finlay D, et al. Correlation of bone scintigraphy and histological findings in medial tibial syndrome. British journal of sports medicine. 2000;34:49-53.

20. Detmer D E. Chronic shin splints. Sports medicine. 1986;3:436-446.

21. Johnell O, Rausing A, Wendeberg B, et al. Morphological bone changes in shin splints. Clinical orthopaedics and related research. 1982;167:180-184.

22. Arendt $E A$, Griffiths $H J$. The use of MR imaging in the assessment and clinical management of stress reactions of bone in high-performance athletes. Clinics in sports medicine. 1997;16:291-306.

23. Beck B R. Tibial stress injuries. Sports medicine. 1998;26:265-279.

24. Winters M, Burr DB, van der Hoeven H, Condon KW, Bellemans J, Moen MH. Microcrack-associated bone remodeling is rarely observed in biopsies from athletes with medial tibial stress syndrome. $\mathrm{J}$ Bone Miner Metab. 2019;37(3):496-502.

25. Schultz L W. A treatment for subluxation of the temporomandibular joint. Journal of the American Medical Association. 1937;109:1032-1035.

26. Banks A R. A rationale for prolotherapy. Journal of Orthopaedic Medicine. 1991;13.

27. Liu Y K, Tipton C M, Matches R D, et al. An in Situ Study of a Sclerosing Solution in Rabbit Medial Collateral Ligaments and Its Junction Strength. Connective tissue research. 1983;11:95-102.

28. Safadi F F, Barbe M F, Abdelmagid S M, et al. Bone structure, development and bone biology. Bone pathology: Springer, 2009. p. 1-50.

29. Scarpone M, Rabago D, Zgierska A, et al. The efficacy of prolotherapy for lateral epicondylosis: a pilot study. Clinical journal of sport medicine: official journal of the Canadian Academy of Sport Medicine. 2008;18:248.

30. Topol G A, Reeves K D, Hassanein K M. Efficacy of dextrose prolotherapy in elite male kicking-sport athletes with chronic groin pain. Archives of physical medicine and rehabilitation. 2005;86:697-702.

31. Ryan M B, Wong A, Gillies J, et al. Sonographically guided intratendinous injections of hyperosmolar dextrose/lidocaine: a pilot study for the treatment of chronic plantar fasciitis. British journal of sports medicine. 2009;43:303-306.

32. Yelland M J, Sweeting K R, Lyftogt J A, et al. Prolotherapy injections and eccentric loading exercises for painful Achilles tendinosis: a randomised trial. British journal of sports medicine. 2011;45:421428. 
33. Khan S, Kumar A, Varshney M, et al. Dextrose prolotherapy for recalcitrant coccygodynia. Journal of Orthopaedic Surgery. 2008;16.

34. Curtin M, Crisp T, Malliaras P, Padhiar N. The effectiveness of prolotherapy in the management of recalcitrant medial tibial stress syndrome: a pilot study. Br J Sports Med 2011;45:e1. doi: 10.1136/bjsm.2010.081554.8

35. Huskisson E. Measurement of pain. The Lancet. 1974;304:1127-1131.

36. Crossley K M, Bennell K L, Cowan S M, et al. Analysis of outcome measures for persons with patellofemoral pain: which are reliable and valid? Archives of physical medicine and rehabilitation. 2004;85:815-822.

37. Likert R. A technique for the measurement of attitudes. Archives of psychology. 1932.

38. Rompe J D, Cacchio A, Furia J P, et al. Low-energy extracorporeal shock wave therapy as a treatment for medial tibial stress syndrome. The American journal of sports medicine. 2010;38:125-132.

39. Moen MH, Schmikli SL, Weir A, et al. A prospective study on MRI findings and prognostic factors in athletes with MTSS. Scand J Med Sci Sports. 2014;24(1):204-210. 40 Lazzarini KM, Troiano TN, Smith RC. Can running cause the appearance of marrow oedema on MR images of the foot and ankle? Radiology. 1997 Feb;202(2):540-2.

40. Bergman AG, Fredericson M, Ho C, Matheson GO. Asymptomatic tibial stress reactions: MRI detection and clinical follow-up in distance runners. AJR Am J Roentgenol. 2004 Sep;183(3):635-8.

41. Winters M, Moen MH, Zimmermann WO, Lindeboom R, Weir A, Backx FG, Bakker EW. The medial tibial stress syndrome score: a new patient-reported outcome measure. Br J Sports Med. 2016 Oct;50(19):1192-9.

42. Nauck T, Lohrer $\mathrm{H}$, Padhiar N, King JB. Development and validation of a questionnaire to measure the severity of functional limitations and reduction of sports ability in German-speaking patients with exercise-induced leg pain. Br J Sports Med. 2015 Jan;49(2):113-7.

43. Korakakis V, Malliaropoulos N, Baliotis K, Papadopoulou S, Padhiar N, Nauck T, Lohrer H. Crosscultural Adaptation and Validation of the Exercise-Induced Leg Pain Questionnaire for English- and Greek-Speaking Individuals. J Orthop Sports Phys Ther. 2015 Jun;45(6):485-96.

\section{Figures}



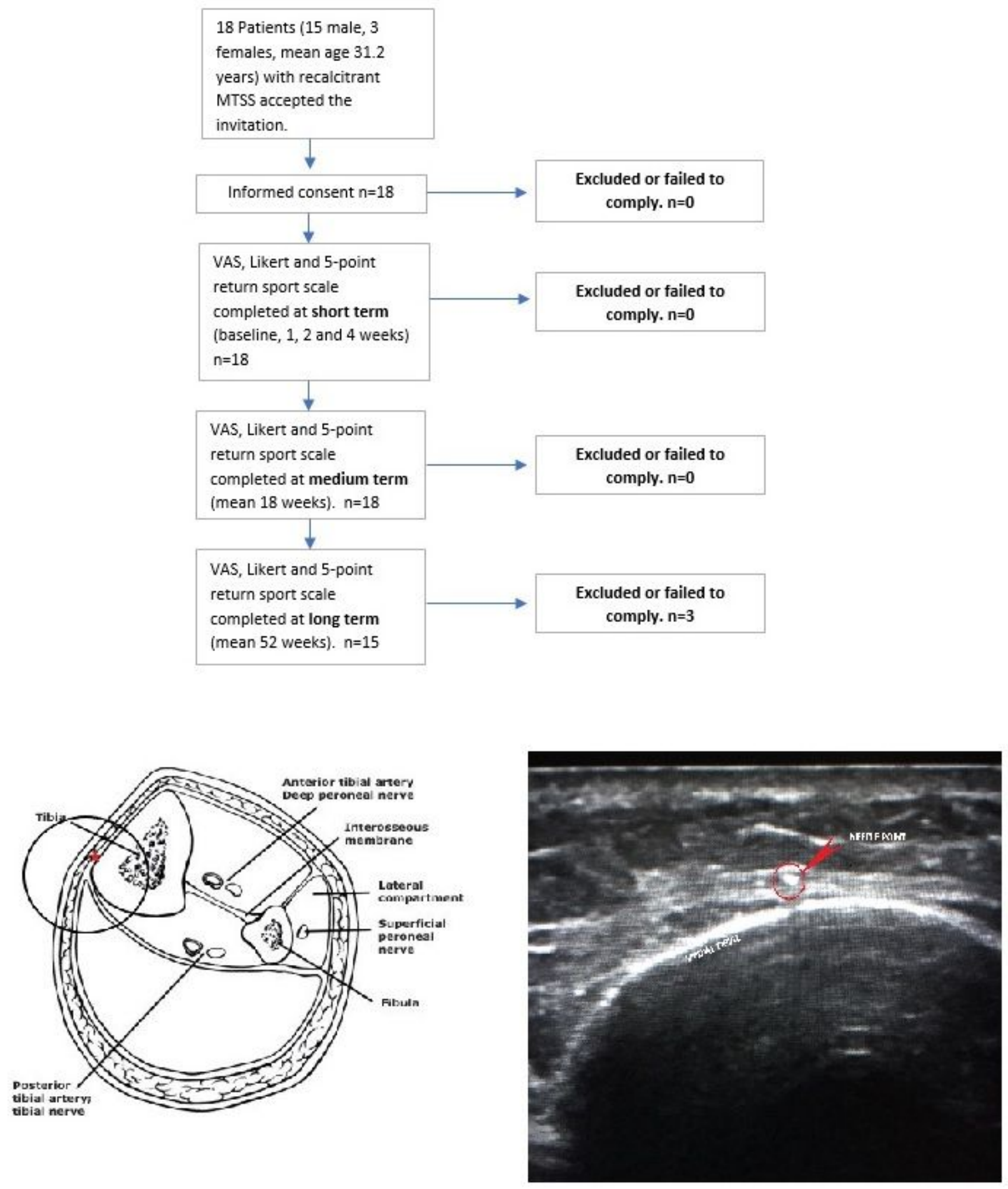

\section{Figure 1}

Recruitment process. A. Cross section diagram of the leg. Red star marks the target area, anterior to deep crural fascia along the medial tibia. Transverse ultrasound image of the needle position (marked with the red circle/arrow). 

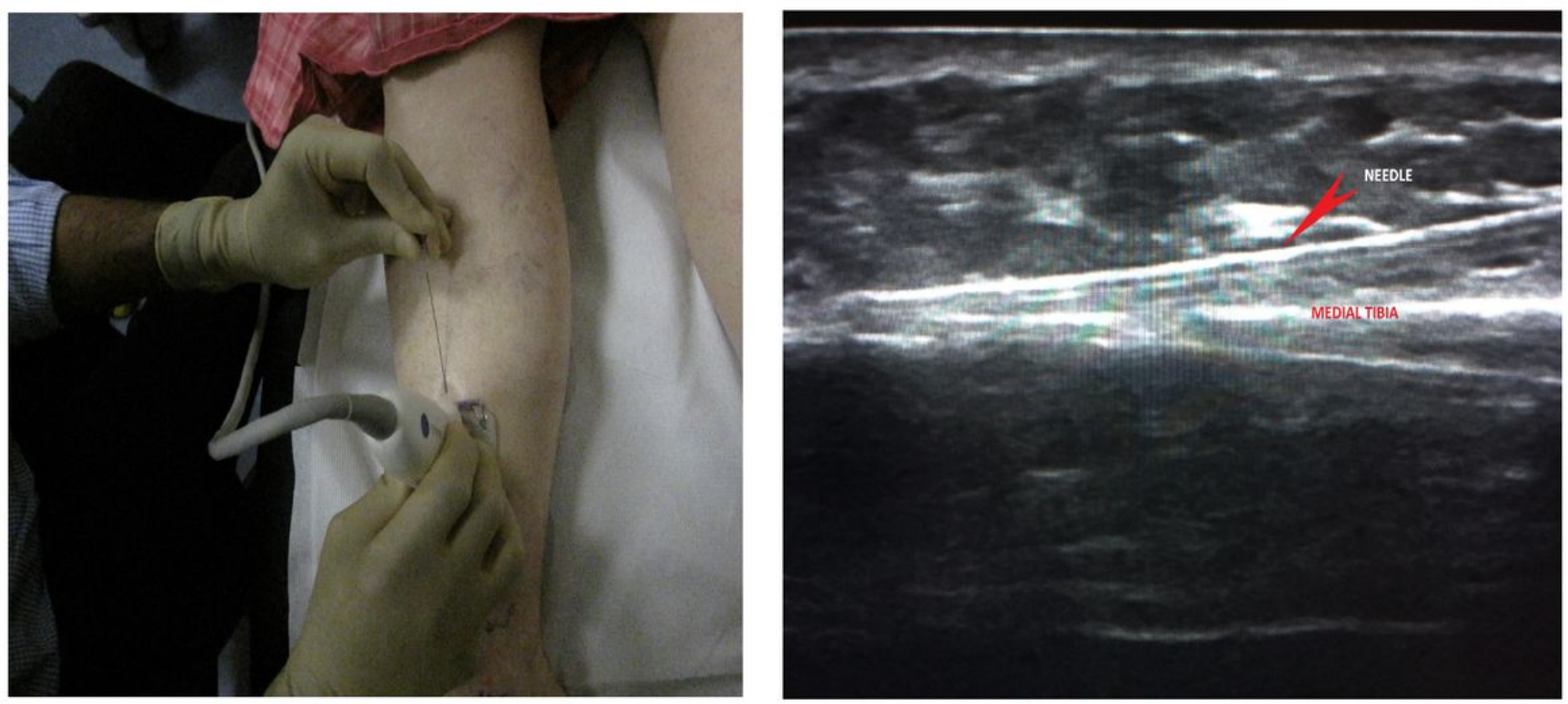

Figure 2

A. The spinal needle was inserted under ultrasound guidance into the medial tibia and just anterior to start of the deep crural fascia region under ultrasound guidance. B. Longitudinal USS image showing the needle position. Please note that initially it is at an angle but final position needs to be parallel with the medial tibia at the target site. 


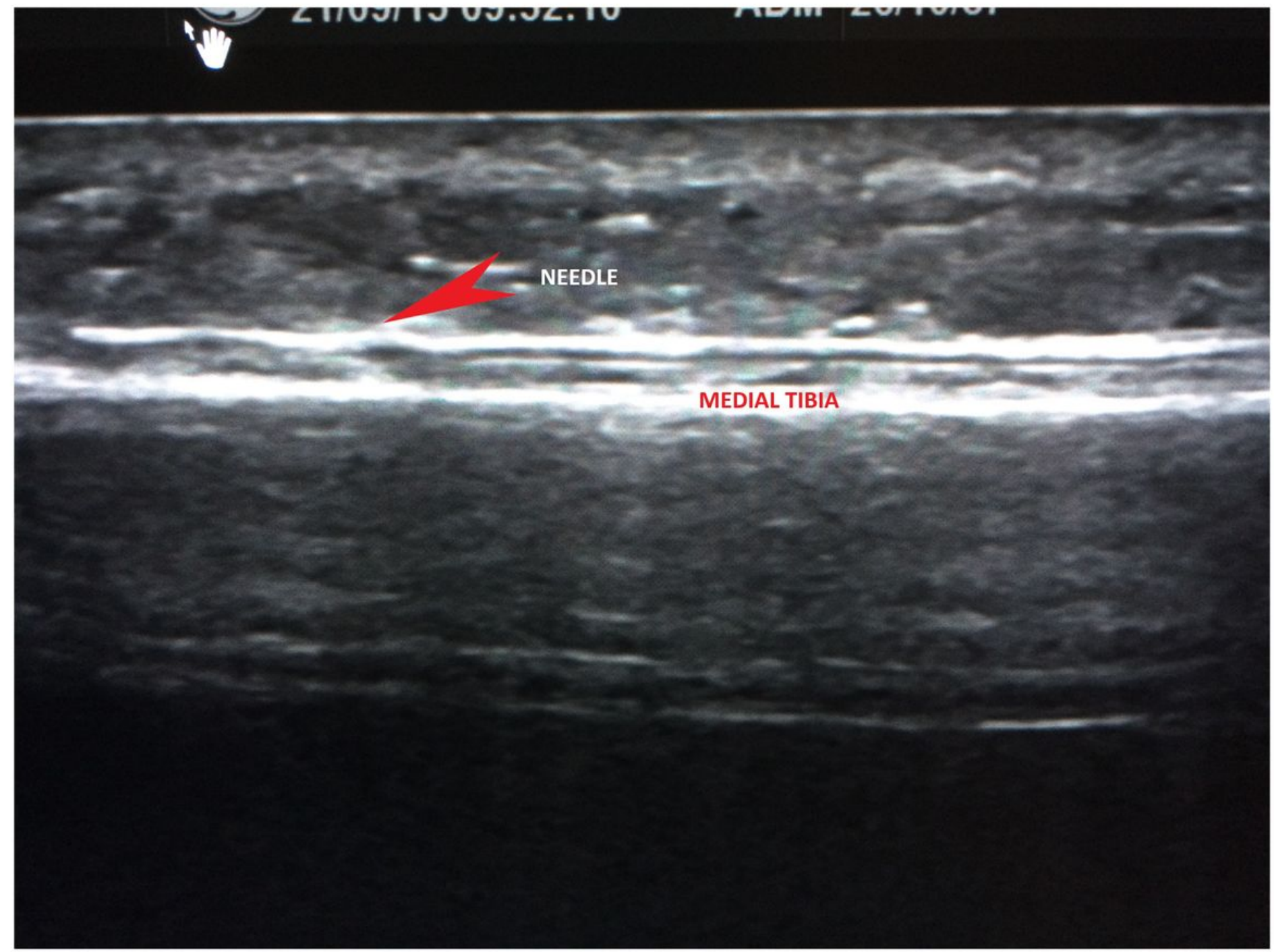

\section{Figure 3}

Longitudinal USS image showing the final needle position which is positioned parallel with the medial tibia at the target site. 


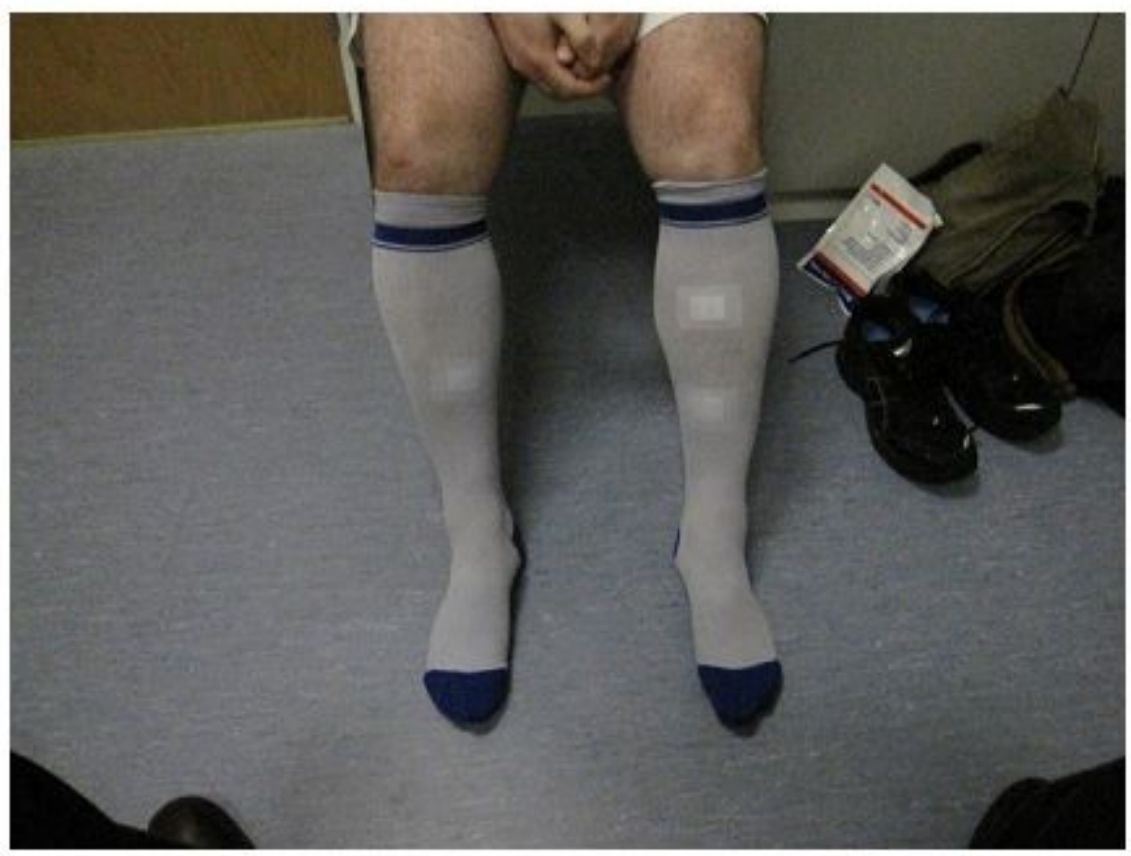

Figure 4

After the injection care involved cleansing the skin, applying wound dressing, ice and compression socks. 


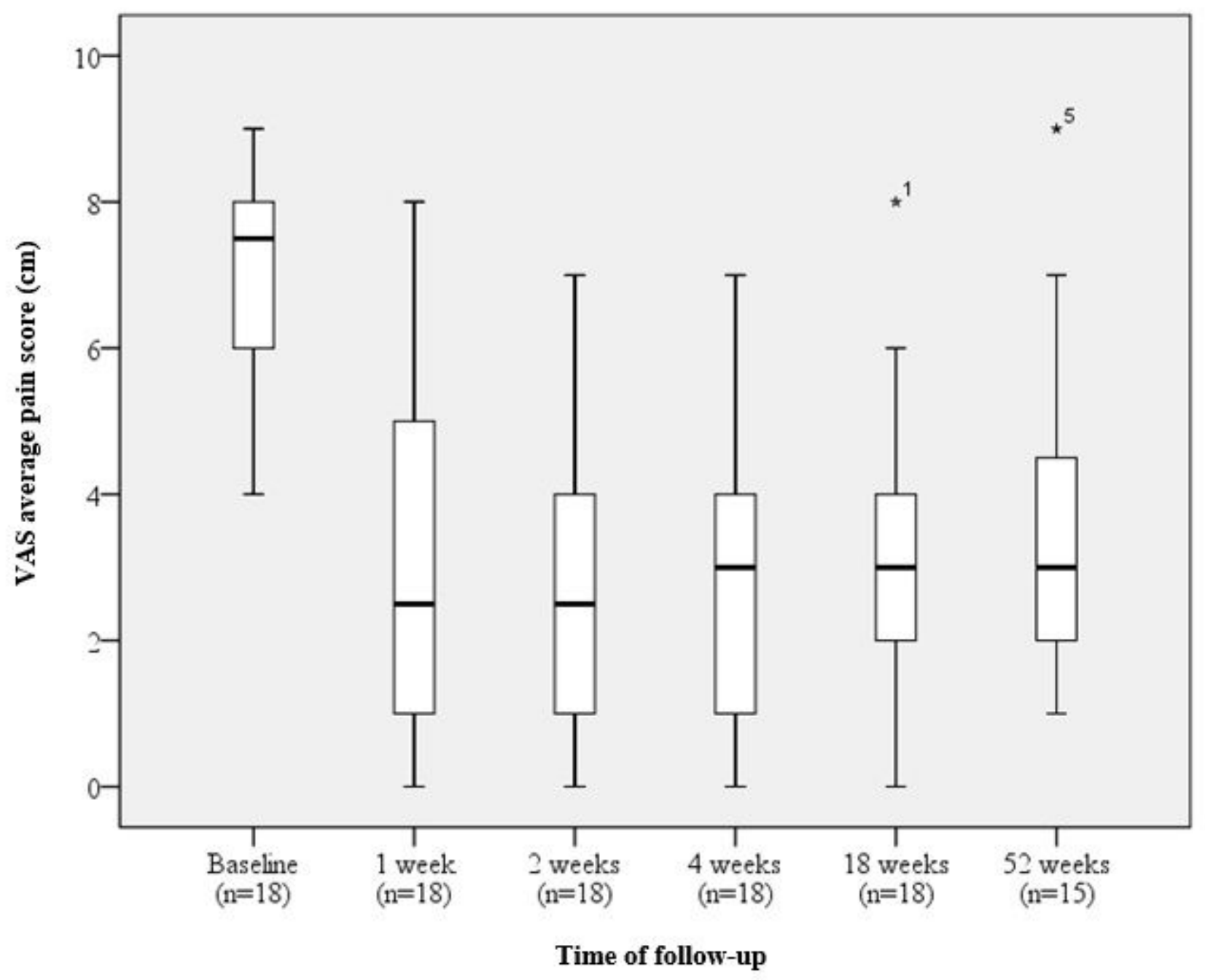

Figure 5

Box plot of median and interquartile range VAS average pain scores for the group of patients at follow-up.

* Indicates a potential outlier (number refers to patient number). 